\title{
Improvement of Drought Tolerance and Grain Yield in Common Bean by Overexpressing Trehalose-6-Phosphate Synthase in Rhizobia
}

\author{
Ramón Suárez, ${ }^{1}$ Arnoldo Wong, ${ }^{1}$ Mario Ramírez, ${ }^{2}$ Aarón Barraza, ${ }^{1}$ María del Carmen Orozco, ${ }^{1}$ \\ Miguel A. Cevallos, ${ }^{2}$ Miguel Lara, ${ }^{2}$ Georgina Hernández, ${ }^{2}$ and Gabriel Iturriaga ${ }^{1}$ \\ ${ }^{1}$ Centro de Investigación en Biotecnología, Universidad Autónoma del Estado de Morelos, Av. Universidad 1001, \\ Col. Chamilpa, Cuernavaca Mor. 62209, Mexico; ${ }^{2}$ Centro de Ciencias Genómicas, Universidad Nacional Autónoma \\ de México, Av. Universidad 2001, Col. Chamilpa, Cuernavaca Mor. 62210, Mexico
}

Submitted 1 November 2007. Accepted 4 March 2008.

\begin{abstract}
Improving stress tolerance and yield in crops are major goals for agriculture. Here, we show a new strategy to increase drought tolerance and yield in legumes by overexpressing trehalose-6-phosphate synthase in the symbiotic bacterium Rhizobium etli. Phaseolus vulgaris (common beans) plants inoculated with $R$. etli overexpressing trehalose-6-phosphate synthase gene had more nodules with increased nitrogenase activity and higher biomass compared with plants inoculated with wild-type $R$. etli. In contrast, plants inoculated with an $R$. etli mutant in trehalose-6phosphate synthase gene had fewer nodules and less nitrogenase activity and biomass. Three-week-old plants subjected to drought stress fully recovered whereas plants inoculated with a wild-type or mutant strain wilted and died. The yield of bean plants inoculated with $R$. etli overexpressing trehalose-6-phosphate synthase gene and grown with constant irrigation increased more than $50 \%$. Macroarray analysis of 7,200 expressed sequence tags from nodules of plants inoculated with the strain overexpressing trehalose-6-phosphate synthase gene revealed upregulation of genes involved in stress tolerance and carbon and nitrogen metabolism, suggesting a signaling mechanism for trehalose. Thus, trehalose metabolism in rhizobia is key for signaling plant growth, yield, and adaptation to abiotic stress, and its manipulation has a major agronomical impact on leguminous plants.
\end{abstract}

Additional keywords: OtsA, otsA $A^{-}$, TPS.

Environmental conditions are severe limiting factors for growth and yield in crops. Among these factors are drought stress, salinity, and extreme temperatures, which dehydrate the plant tissues and cause irreversible cellular damage and death (Bartels and Sunkar 2005; Bray et al. 2000). However, there are some organisms that can withstand long periods in a dried state and have the capacity to rehydrate and restart their metabolic functions after being in contact with water (Clegg 2001; Crowe et al. 1992). These anhydrobiotic organisms are rare; among them are resurrection plants such as Selaginella lepidophylla and some fungi such as Saccharomyces cerevisiae. All these organisms have in common the capacity of synthesizing

Corresponding author: Gabriel Iturriaga; E-mail: iturri@buzon.uaem.mx.

Data deposition: GenBank accession number AY731700. and accumulating large concentrations of trehalose (Elbein et al. 2003). This is a nonreducing disaccharide ( $\alpha$-D-glucopyranosyl-1, 1- $\alpha$-D-glucopyranoside) formed by two molecules of glucose linked through their anomeric carbons.

There are five different pathways for trehalose biosynthesis; the most common is a two-step process involving glucose transfer from UDP-glucose to glucose-6-phosphate to form trehalose-6-phosphate and UDP (Avonce et al. 2006). This reaction is catalyzed by the enzyme trehalose-6-phosphate synthase (TPS) encoded by the OtsA gene in bacteria (Ström and Kaasen 1993). The second step results from the dephosphorylation of trehalose-6-phosphate and is catalyzed by trehalose-6-phosphate phosphatase (TPP). The enzyme trehalase degrades trehalose into two molecules of $\alpha$-D-glucose and is present in most organisms (Aeschbacher et al. 1999). Trehalose in bacteria dramatically accumulates in osmotic or thermal stress conditions. Experiments done in Escherichia coli showed that mutation of OtsA restricts its capacity to grow in stress conditions (Giaever et al. 1988; Kandror et al. 2002).

Symbiotic nitrogen-fixing bacteria such as Rhizobium and related genera have the capacity of synthesizing trehalose, and its accumulation has been detected in bacteroids as well as in nodules (Müller et al. 2001; Streeter 1985). Rhizobium etli, a symbiont of Phaseolus vulgaris, has the capacity to induce nodule formation on the roots of bean plants (Sessitsch et al. 2002). Recently, the full genome sequence of $R$. etli has been reported (González et al. 2006).

Bean crops (P. vulgaris) are a staple food of great economic importance around the world, in particular in developing countries; nevertheless, their growth is affected by climatic conditions restricting grain yield (Subbarao et al. 1995). Mineral nitrogen often limits plant growth, and agriculture and food supplies largely depend on the nitrogen cycle. Although nitrogenous fertilizers have allowed the expansion of crop yields, they have dramatically contributed to air and water pollution, acid rain, and the greenhouse effect (Socolow 1999). Symbiotic relationships have evolved the capacity to fix atmospheric nitrogen in the rhizobia-colonizing legume nodules and are responsible for converting it to 70 million tons of ammonia each year (Brockwell et al. 1995).

To gain understanding of the role of trehalose metabolism in rhizobia, we overexpressed the OtsA gene in $R$. etli, isolated an insertion mutant in the same gene, and analyzed the effect on free-living bacteria and symbiotic bacteroids at the molecular, biochemical, and physiological levels. Here, we show that common bean plants inoculated with $R$. etli overexpressing 
OtsA are stress tolerant, and that there is a substantial increase in grain yield.

\section{RESULTS}

The sequence of the OtsA gene from $R$. etli (ReOtsA) revealed an open reading frame (ORF) encoding a 469-aminoacid deduced protein. It showed significant sequence identity with other genes encoding TPS, such as $74 \%$ to Sinorhizobium meliloti and $33 \%$ to Saccharomyces cerevisae homologs at the amino acid level. The full-length gene sequence of 1,621 bp includes the $5^{\prime}$ and $3^{\prime}$ untranslated regions. To overexpress the ReOtsA gene, we used the broad-host-range pBBR1MCS5 vector that allows gene expression under the control of the lac promoter (Kovach et al. 1995). The ReOtsA 1.4-kb ORF was amplified by polymerase chain reaction (PCR), subcloned into this vector, and mobilized to $R$. etli. In addition, an insertion mutant in the OtsA gene was isolated at a frequency of $1 \times 10^{-9}$ by homologous recombination after knocking out the OtsA gene with a tetracycline resistant marker.

The $R$. etli wild-type (WT), OtsA mutant (ots $\left.A^{-}\right)$, and overexpressing $O t s A(\mathrm{Ox})$ strains were grown in minimal medium for 2 days. After $24 \mathrm{~h}$, the Ox strain continued growing, whereas the WT and $o t s A^{-}$started to decline (Fig. 1A). When the three strains were grown in the presence of $0.5 \mathrm{M} \mathrm{NaCl}$, the Ox strain grew at a significantly higher rate than the WT and ots $A^{-}$strains (Fig. 1B). In fact, growth of the ots $A^{-}$mutant in $0.5 \mathrm{M} \mathrm{NaCl}$ was negligible. The Ox strain also survived better than WT and ots $A^{-}$strains if exponentially grown cells were heat shocked or freeze thawed before plating (data not shown). These results indicate that the overexpression of the $O t s A$ gene provides stress tolerance to $R$. etli. Trehalose quantification was carried in ots $A^{-}$, Ox, and WT strains grown in media without osmotic stress (Fig. 1C) or containing $0.5 \mathrm{M}$ $\mathrm{NaCl}$ (Fig. 1D). There was a higher trehalose concentration in Ox cells compared with WT due to the expression of ReOtsA driven by the constitutive lac promoter, whereas the ots $A^{-}$ strain has a lower concentration of the disaccharide (Fig. 1C). The trehalose content in the Ox strain was significantly higher after $24 \mathrm{~h}$ of growth in the presence of $\mathrm{NaCl}$, suggesting a stress induction of $O t s B$ (encodes for TPP) and possibly of the other trehalose biosynthetic genes that we found in the $R$. etli genome after a bioinformatics analysis. These genes are a ReOtsA homolog which shares $45 \%$ identity and $60 \%$ similarity at the amino acid level, and genes of other trehalose biosynthetic pathways, namely two encoding trehalose synthase which converts maltose into trehalose and one gene encoding a maltooligosyl trehalose synthase that synthesizes trehalose from maltooligosaccharides (Avonce et al. 2006). The mutant strain also had a slight increase in trehalose content upon stress but at a lower level compared with the WT strain. Thus, the trehalose concentration correlated with improved stress tolerance in free-living $R$. etli cells.

In order to determine the effect of the overexpression or mutation of the OtsA gene in $R$. etli in symbiosis with nodulating $P$. vulgaris, 2-day-old bean plantlets were inoculated with the WT, Ox, or ots $A^{-}$strains and allowed to grow up to 21 days post inoculation (dpi). Plants nodulated with the Ox strain showed a $27 \%$ increase in nodule number compared with the WT, whereas a decrease of $26 \%$ was observed in bean plants inoculated with the ots $A^{-}$mutant (Fig. 2A). To gain further understanding at the biochemical level, the nitrogenase activity was determined as acetylene reduction activity by gas chromatography analysis from nodules of plants inoculated with the three strains. Plants nodulated with the Ox strain showed a $38 \%$ increase in nitrogenase activity, whereas a $45 \%$ decrease in activity was observed in plants inoculated with the ots $A^{-}$
A
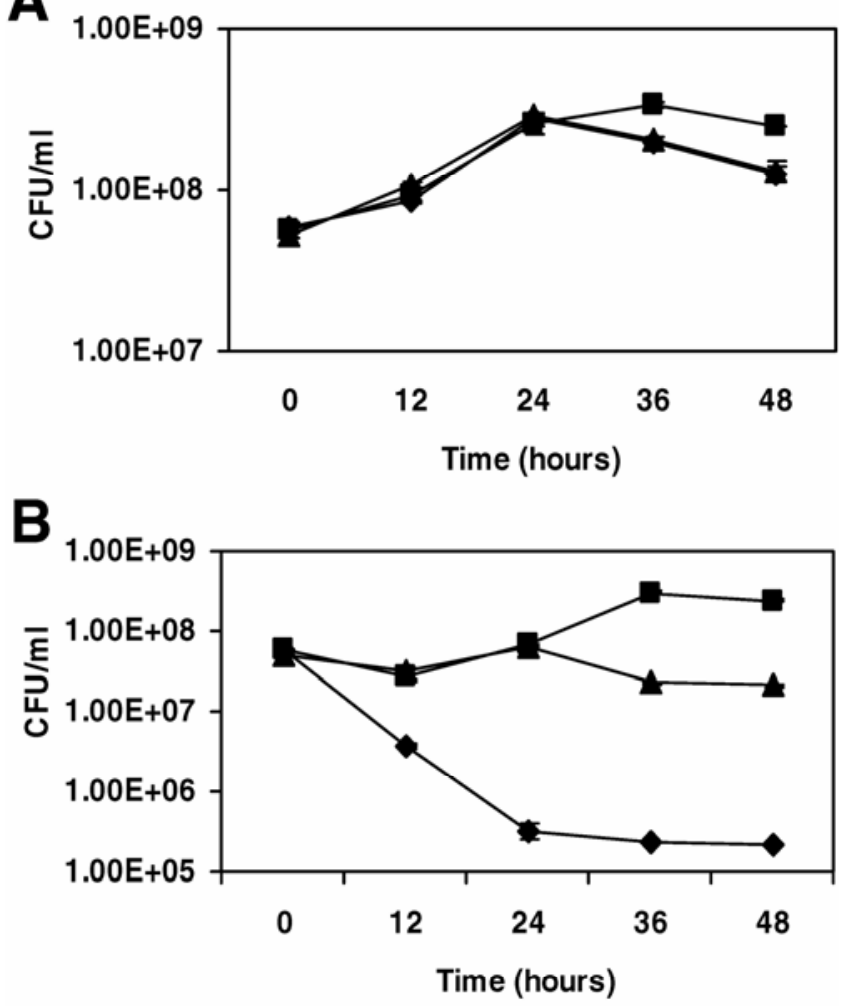

C

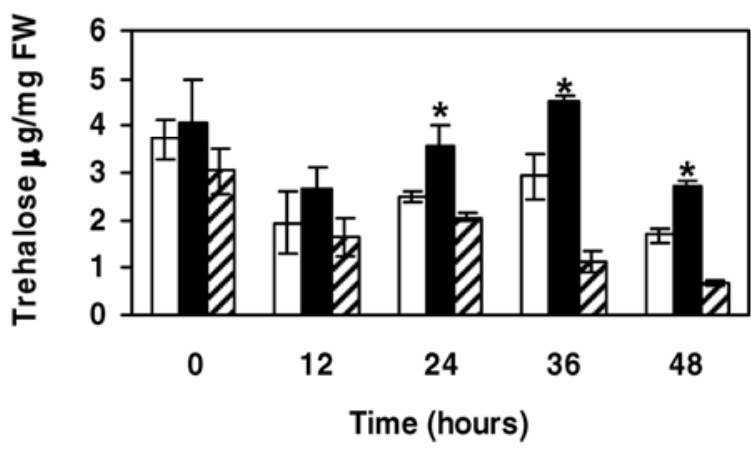

D

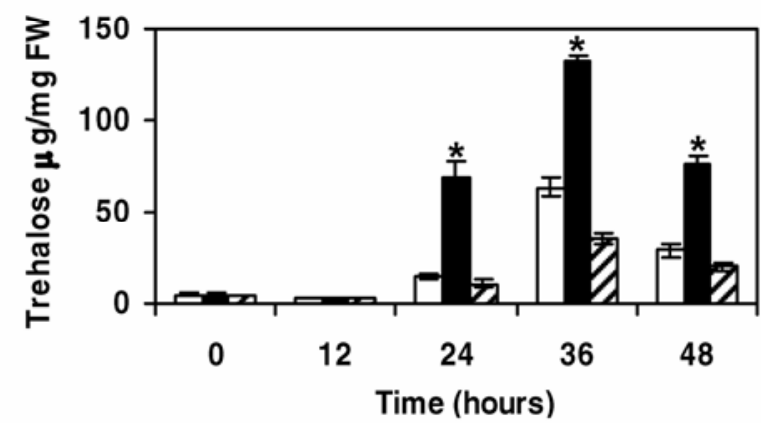

Fig. 1. Survival of free-living Rhizobium etli with overexpressed or mutated OtsA gene upon stress. Wild-type (triangles), overexpressing OtsA gene (squares), and ots $A^{-}$mutant (rhombus) strains of $R$. etli were grown at $30^{\circ} \mathrm{C}$ in $\mathbf{A}$, liquid minimal medium alone or $\mathbf{B}$, containing $0.5 \mathrm{M} \mathrm{NaCl}$. At the indicated times, equal amounts of cells were plated on petri dishes, transferred to $30^{\circ} \mathrm{C}$, and grown for $48 \mathrm{~h}$ before surviving colonies were counted. Wild-type (white bars), overexpressing OtsA gene (black bars), and $o t s A^{-}$mutant (gray bars) strains of $R$. etli were grown as described before, and aliquots were taken to measure intracellular trehalose content from the same cultures used to count viable cells in $\mathbf{C}$, minimal medium alone or $\mathbf{D}$, containing $0.5 \mathrm{M} \mathrm{NaCl}$. Both figures represent the results of three independent experiments. Asterisks indicate that the means of the samples are different at the 0.05 level $(P<0.05)$. 
mutant (Fig. 2B). Histological analysis of nodules from constantly irrigated (Fig. 2C) and drought-stressed plants (Fig. 2D) showed a larger area of infected cells in the nodules of plants inoculated with the $R$. etli Ox strain and smaller infected area in nodules of plants inoculated with ots $A^{-}$mutant after comparison with the WT strain. All nodules from stressed plants shrank and, thus, look smaller than nodules from unstressed plants (Fig. 2C and D).

A major objective in the present work was to determine whether overexpression of the OtsA gene of $R$. etli improves tolerance to drought stress of inoculated bean plants. Plants inoculated with WT, Ox, or ots $A^{-}$strains were grown with continuous watering for 21 days. Afterward, watering was stopped for 3 weeks and then watering was resumed. Plants inoculated with the Ox strain showed evident drought tolerance compared with those inoculated with the WT or ots $A^{-}$strains that did not survive stress (Fig. 3A). Two days after watering was resumed, $87 \%$ of the plants inoculated with $R$. etli overexpressing the OtsA gene had the capacity for recovering from drought stress whereas only $7 \%$ of plants inoculated with the WT strain and less than $1 \%$ of plants inoculated with the $o t s A^{-}$mutant survived (Fig. 3B). The relative water content (RWC) of the plants and soil gravimetric water content (SGWC) were determined during the experimental time. At $21 \mathrm{dpi}$, just before drought stress was applied, RWC and SGWC were at their highest values (taken as 100\%) and fairly equal for all plants (Fig. 3C and D). However, at 42 dpi that corresponded to 3 weeks of water deprivation, plants lost approximately $90 \%$ of their water content. There was a slightly higher RWC in the bean plants inoculated with the Ox strain $(14.7 \%)$ compared with plants inoculated

A

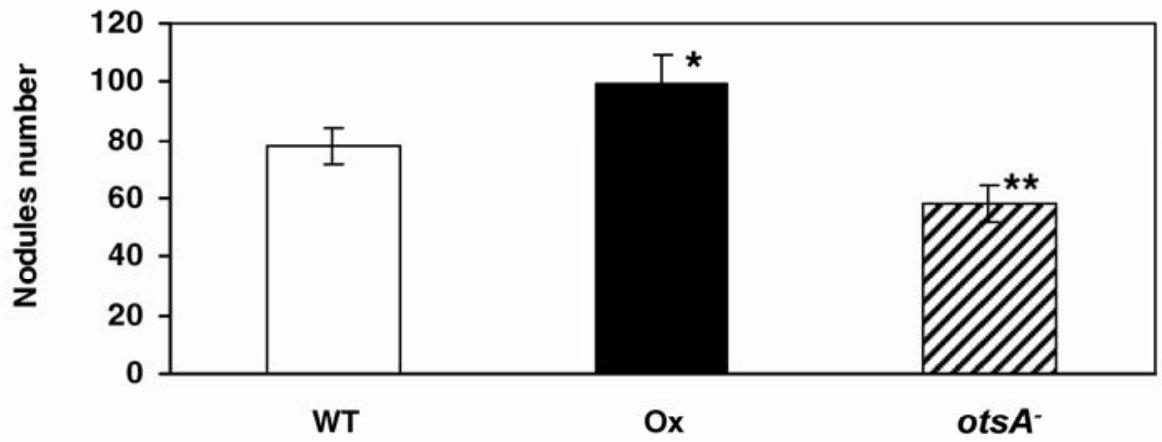

B

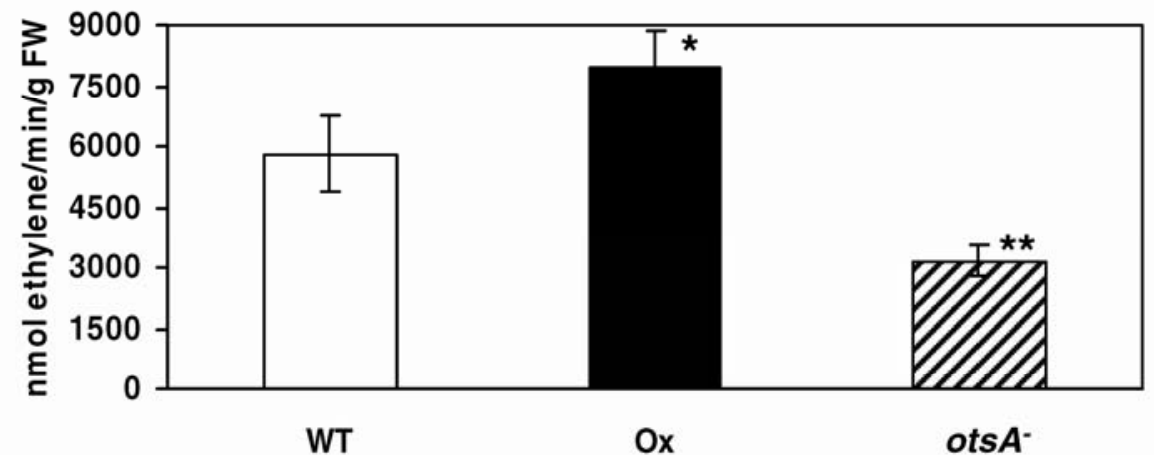

C

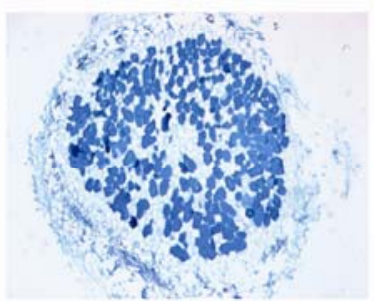

WT

D

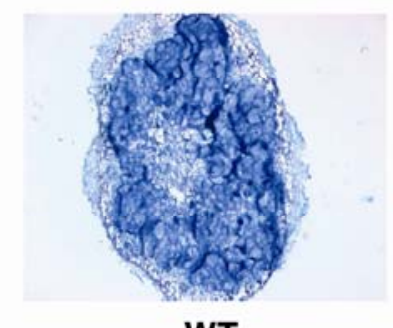

WT

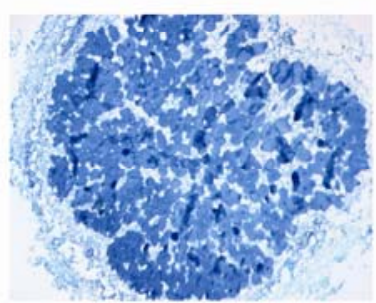

Ox

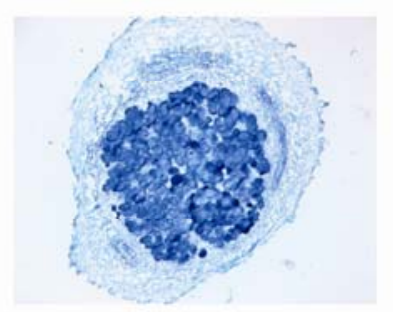

Ox

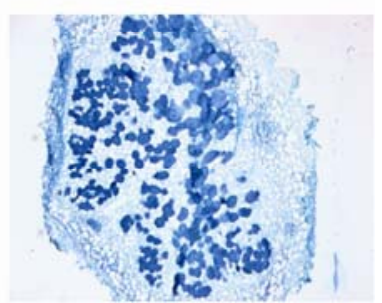

ots $A^{-}$

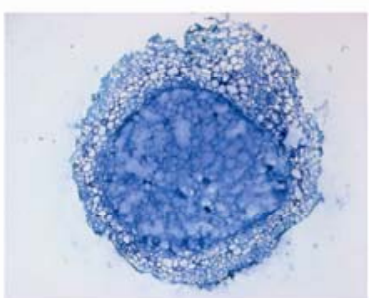

otsA-

Fig. 2. Biochemical and histological analyses of bean nodules. A, Number of nodules and B, nitrogenase activity were determined from common bean plants inoculated with the wild type (WT), R. etli strain overexpressing the OtsA gene (Ox), or OtsA mutant (ots $A^{-}$). These data represent the results of three independent experiments. Bright-field photographs of transversal sections from $\mathbf{C}$, a representative nodule of irrigated plants or $\mathbf{D}$, drought-stressed plants inoculated with either strain. Single asterisks indicate that the means of the samples are different from WT, and double asterisks indicate that means of the samples are different from WT and Ox, at the 0.05 level $(P<0.05)$. 
with $R$. etli WT $(9.64 \%)$ or ots $A^{-}(9.20 \%)$ strain after drought stress. The SGWC also dropped to approximately $25 \%$ after 21 days without watering.

The quantification of trehalose content in plants revealed significantly higher levels of the disaccharide in nodules than in shoots. In fact, trehalose increased two times when plants were subjected to drought stress (Fig. 3E). However, an increase of approximately $10 \%$ in the disaccharide content was observed in nodules of plants inoculated with the Ox strain compared with nodules inoculated with the WT. In contrast, the trehalose levels decreased significantly in nodules of plants inoculated with the ots $A^{-}$mutant (Fig. 3E). No significant differences were detected in trehalose concentration in shoots of plants inoculated with the three different $R$. etli strains. The disaccharide was not detected in roots free of nodules of plants inoculated with either strain.

The biomass of plants inoculated with the Ox strain was higher than of plants inoculated with WT and lower for plants inoculated with ots $A^{-}$, after measuring both fresh and dry weight (Fig. 4A and B). There was a sharper difference considering constant watering conditions, indicating that the overexpression of ReOtsA provoked up to $92 \%$ of water retention, whereas the ots $A^{-}$mutant led to a $15 \%$ drop of water retention compared with the WT (Fig. 4A). Upon drought stress, the biomass of all plants inoculated with either strain decreased significantly; however, the differences still correlated to the corresponding strain as shown before (Fig. 4A and B).

An interesting question was whether the overexpression or mutation of the OtsA gene in $R$. etli interacting with bean plants would have an energetic cost for the plant, reflected in the grain yield. Therefore, three groups of 10 plants each were inoculated with the WT, Ox, or ots $A^{-}$strains of $R$. etli and kept under continuous watering until obtaining mature seed. The yield (see Material and Methods), which considered number of pods (legume fruits) and seed and their dry weight, showed an increase of $57 \%$ in plants inoculated with the Ox strain compared with plants nodulated with the WT strain, whereas a drop of $43 \%$ in yield was observed in plants inoculated with the ots $A^{-}$mutant, when plants were constantly irrigated (Fig. 4C). Upon drought stress, the yield sharply decreased but was still $38 \%$ higher in plants inoculated with the Ox strain and negligible for plants nodulated with the ots $A^{-}$mutant (Fig. 4C).

The overexpression of a TPS gene in Arabidopsis leads to an altered gene-expression profile, and it has been suggested a signaling role for trehalose and trehalose-6-phosphate (Avonce et al. 2004; Schluepmann et al. 2004). To explore if the changes in yield and stress tolerance observed in common bean inoculated with either Ox or ots $A^{-}$strains were due to a change in the plant gene-expression pattern, a macroarray

Fig. 3. Survival of plants subjected to drought stress. Well-watered, 3week-old common bean plants were subjected to drought stress by withholding watering for another 3 weeks. In each pot, five plants inoculated with Rhizobium etli wild-type (WT), overexpressing the OtsA gene (Ox), or ots $A^{-}$strain were present. A, Photographs were taken 2 days after rewatering. Survival of imposed drought stress on plants nodulated with either strain was determined considering 15 plants of each condition, and three independent experiments were conducted. B, Survival of plants upon continual watering was taken as $100 \%$. C, Relative water content (RWC) and D, soil gravimetric water content (SGWC) were determined before (21 days postinoculation [dpi]) and after (42 dpi) water stress. Trehalose was determined in shoots and nodules from plants nodulated with the WT, Ox, or ots $^{-}$strain after 3 weeks of drought treatment or $\mathbf{E}$, continual watering. Asterisks indicate that the means of the samples are different at the 0.05 level $(P<0.05)$. approach was used. Nylon filter arrays with a redundant set of 7,200 expressed sequence tags (EST), containing 1,806 unique genes, from a $P$. vulgaris nodule cDNA library (Ramírez et al. 2005) were hybridized with cDNA from total RNA nodules of bean plants inoculated with either the $R$. etli WT, Ox, or ots $A^{-}$ strain. After hybridization, the signal intensity of each spot was quantified and the expression ratio of each gene in nod-
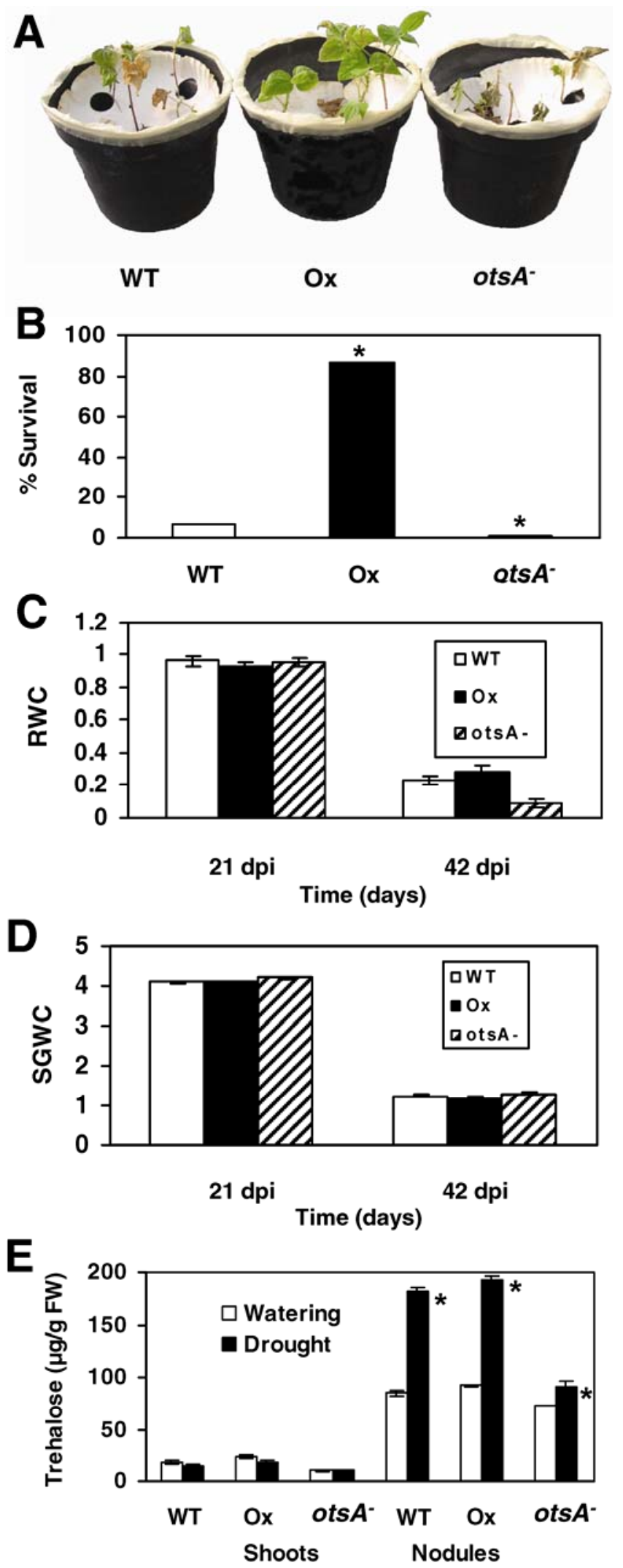

Vol. 21, No. 7, 2008 / 961 
ules of plants inoculated with either the Ox or ots $A^{-}$strain compared with WT-inoculated plants was calculated. Such comparison was done in $P$. vulgaris plants subjected to drought stress and in continually irrigated plants. Overall, 3,485 EST were upregulated in plants subjected to drought stress whereas only 177 EST were induced in plants under continual irrigation, after comparing the ratio of genes induced in plants inoculated with Ox versus WT. Among the most highly upregulated genes in nodules of Ox-inoculated plants were those involved in nodule function, nitrogen metabolism, tolerance to abiotic stress, and carbon metabolism; the expression ratios of some of these genes are shown in Table 1.

The analyzed genes were highly induced in nodules of drought-stressed plants inoculated with the Ox strain compared with plants inoculated with the WT strain, showing expression ratios over 30 -fold. In the irrigated plants, only some genes showed upregulation in Ox-elicited nodules, with lower ex-
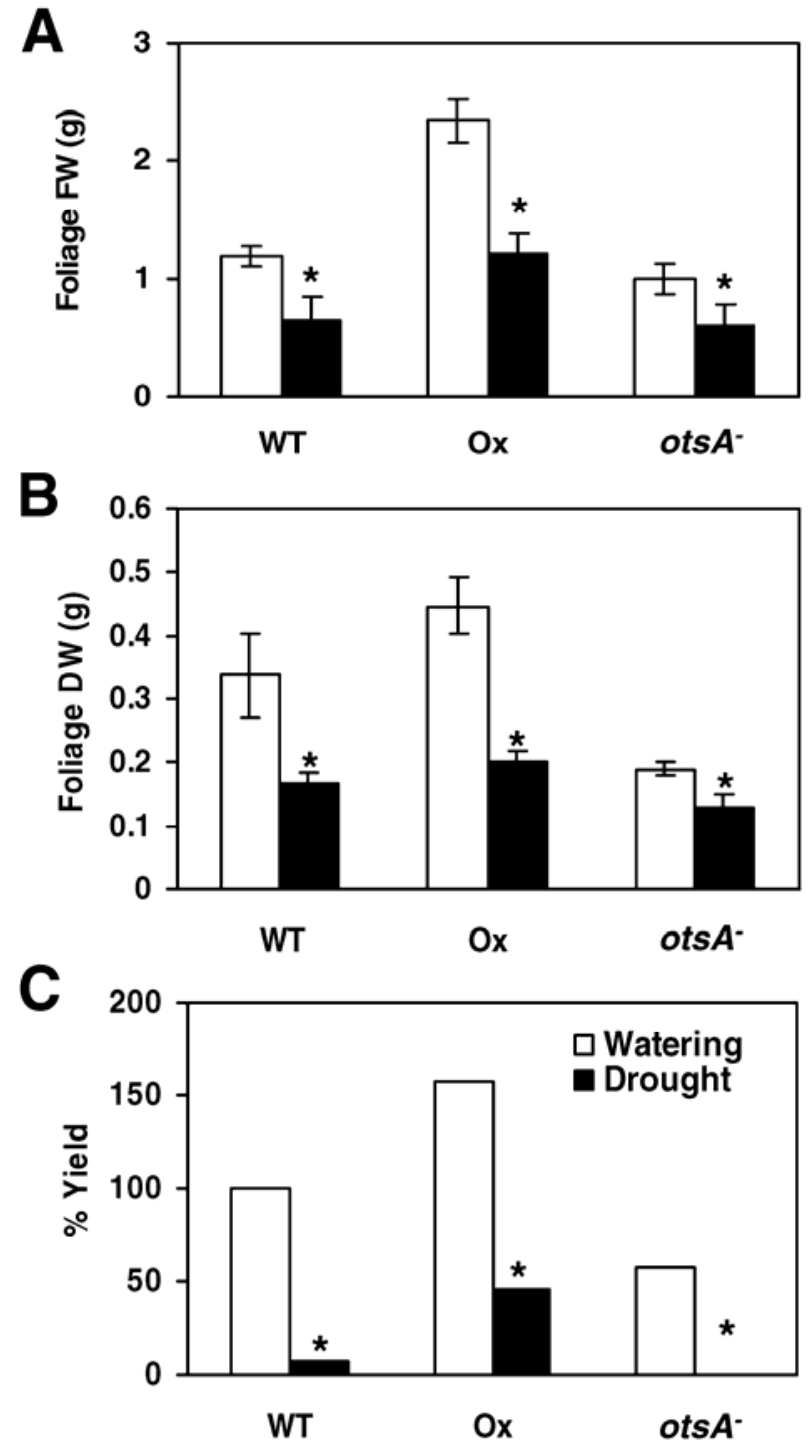

Fig. 4. Plant biomass and grain yield. A, Foliage fresh weight or $\mathbf{B}$, dry weight of bean plants at 21 days after inoculation with Rhizobium etli wild-type (WT), overexpressing the OtsA gene (Ox) or ots $A^{-}$strains was determined. C, Grain yield in Phaseolus vulgaris plants nodulated with WT, Ox, or ots $A^{-}$strains was determined in three independent experiments. Percentages were calculated taking the value of the WT strain as $100 \%$. White bars denote continually irrigated plants and black bars plants subjected to drought stress Asterisks indicate that the means of the samples are different at the 0.05 level $(P<0.05)$. pression ratios compared with WT nodules. Genes that are highly expressed in nodules are widely represented in the nodule cDNA library and macroarray used (Ramírez et al. 2005); in agreement, several EST from these contigs show high Ox/WT expression ratios (Table 1). The most conspicuous transcripts highly induced in nodules elicited by $\mathrm{Ox}$ strain were those that encoded leghemoglobin 2, which transports oxygen to the bacteroid; nodulin 30 transcript, so far of unknown function; glutamine synthetase, involved in $\mathrm{NH}_{4}$ assimilation; uricase II, which synthesizes ureids; sucrose synthase, PEP carboxylase, and malate dehydrogenase, which are involved in producing carbon skeletons to feed the symbiotic bacteria; and several detoxification enzymes such as homoglutathione synthetase, catalase, cytochrome P450, glutaredoxin, and ascorbate peroxidase are key to helping plants recover from drought stress (Table 1). None of the analyzed genes were induced in nodules from plants inoculated with the ots $A^{-}$ mutant strain, whether irrigated or subjected to drought stress (data not shown).

\section{DISCUSSION}

Breeding has had limited success in improving drought tolerance in crops due to the multigenic nature of the trait. A more promising strategy is the overexpression of genes conferring tolerance to abiotic stress. Trehalose is involved in stress tolerance in ahydrobiotic organisms and in transgenic animals and plants. In the present work, the overexpression and mutation of the ReOtsA gene in $R$. etli was evaluated in both freeliving and symbiotic conditions.

We showed that free-living $R$. etli has a significantly higher survival capacity to osmotic stress when the bacteria overexpressed ReOtsA (Fig. 1). Similarly, the overexpression of OtsAB genes in E. coli increased tolerance to osmotic stress (Purvis et al. 2005). Addition of exogenous trehalose to Bradyrhizobium japonicum confers tolerance to dehydration stress (Streeter 2003). In contrast, mutation of ReOtsA impaired stress tolerance (Fig. 1). However, we still could detect trehalose in the ots $A^{-}$ mutant, although at relatively lower levels compared with the WT strain. This is probably due to the presence of three trehalose biosynthetic pathways in $R$. etli that we found by bioinformatics analysis: an OtsA homolog that encodes TPS, a trehalose synthase gene, and a trehalose maltooligosyl trehalose synthase gene. Interestingly, these same three routes have been found in B. japonicum as well, and their significant transcription induction correlates with an elevated level of trehalose, suggesting an important role of this disaccharide in desiccation tolerance (Cytryn et al. 2007). Another striking result is the hyperaccumulation of trehalose when the Ox strain was subjected to osmotic stress (Fig. 1). This could be explained by the stress induction of $O t s B$, the OtsA homolog, or the other trehalose biosynthetic genes present in the $R$. etli genome.

We also examined the effects of overexpressing or mutating ReOtsA in $R$. etli in symbiosis with $P$. vulgaris and, for this aim, several parameters were evaluated. First, a higher number of nodules and nitrogenase activity were found in plants inoculated with the Ox strain, whereas a lower amount of both were present in plants inoculated with the ots $A^{-}$mutant strain (Fig. 2). Also, we have shown that the expression of the leghemoglobin 2 gene in nodules from plants inoculated with the Ox strain was increased significantly, suggesting a possible increase in bacteroid respiration and, thus, a higher nitrogenase activity and nitrogen fixation. Trehalose might also protect nitrogenase from the inactivation caused by oxygen. It has been shown that trehalose has an antioxidant effect (Gonzalez-Parraga et al. 2003).

The increase in nodule number and nitrogenase activity in $P$. vulgaris plants overexpressing ReOtsA suggested that bacterial 
trehalose triggered a morphological and biochemical response leading to a higher biological nitrogen fixation. Therefore, we analyzed a possible effect at the subcellular level in nodules. There was a greater quantity of infected nodule cells in plants inoculated with the Ox strain (Fig. 2). These results could be explained because the accumulation of trehalose in this strain provides it with a greater capacity to infect or to escape the defense mechanisms in nodulating plants, thus inducing the differentiation of a greater number of cells containing bacteroids. It has been reported that the mutation of the OtsA gene of Mycobacterium tuberculosis causes a decrease in the infectivity of mice (Murphy et al. 2005). Similarly, a $\Delta t p s 1$ mutant in the rice blast fungus Magnaporthe grisea was greatly attenuated in pathogenicity (Foster et al. 2003). Moreover, Tps1 in $M$. grisea plays a central role in the establishment of plant disease (Wilson et al. 2007). Recently, it has been shown that trehalose catabolism in Sinorhizobium meliloti plays a critical role in alfalfa root colonization (Jensen et al. 2005). Therefore, trehalose seems to be involved in the animal- and plantmicroorganism interactions.

One of the essential questions of this work was to determine whether the overexpression of ReOtsA gene in R. etli in symbiosis with common bean plants caused an increase in drought tolerance. In fact, $P$. vulgaris plants inoculated with the $\mathrm{Ox}$ strain had a significant increase in drought tolerance compared with plants inoculated with the WT strain (Fig. 3). As far as we know, this is the first report of the improvement to drought stress of a legume species by a different method than plant breeding or plant transformation.

It is noteworthy that plants inoculated with the Ox strain showed an increase of approximately $10 \%$ in trehalose levels in nodules and leaves. Similar small increases of trehalose have been observed in other instances where a TPS gene was overexpressed in transgenic tobacco, potato, rice, Arabidopsis, or animal cells, and still there was an enhanced drought tolerance (Avonce et al. 2004; Garg et al. 2002; Guo et al. 2000; Holmström et al. 1996; Yeo et al. 2000). The trehalose concentration found was too low to explain an effect of trehalose as osmoprotector (Gaff 1996). Thus, one possibility is that treha- lose could be functioning as a signal molecule activating stress tolerance pathways in the plant (Paul 2007). It is well established that sugars signal plant growth, development, and stress responses, with hexokinase (HXK) having a central role as a glucose sensor (Rolland et al. 2006). In Arabidopsis, a tps 1 knock-out is developmentally arrested in the torpedo stage, which is associated with initiation of storage reserve accumulation (Eastmond et al. 2002). Alterations of trehalose-6-phosphate levels have demonstrated that this metabolic intermediate is indispensable for carbohydrate utilization for plant growth (Schluepmann et al. 2003). Arabidopsis plants overexpressing AtTPS1 are drought-tolerant and insensitive to glucose and ABA, and showed an altered expression of $H X K 1$ and ABI4 genes (Avonce et al. 2004). Recently, the maize ramosa mutant with abnormal inflorescences and lower grain yield was shown to be knocked-out in a TPP gene demonstrating the role of a trehalose metabolic enzyme in development (SatohNagasawa et al. 2006). All these results strongly support the role of trehalose as a signal molecule.

The possible trehalose transport from rhizobia to the nodule plant cells still needs to be demonstrated. However if the latter occurs, we propose that trehalose may be acting as a signal molecule to activate transcription in nodule cells of genes involved in stress tolerance and carbon and nitrogen metabolism (Table 1). Interestingly, in this study, an unexpected link between trehalose metabolism in Rhizobium spp. and plant yield was found: plants inoculated with the overexpressing strain showed up to $57 \%$ improvement in the grain yield (Fig. 4). These results are important because they demonstrate that the overexpression of OtsA gene in $R$. etli increases crop yield. The increase in biomass and grain yield of plants inoculated with $R$. etli overexpressing the OtsA gene is also a consequence of a higher nitrogen fixation because several genes related to this process are upregulated (Table 1). For instance, the leghemoglobin 2 was the highest upregulated gene when bean plants were inoculated with the overexpressing strain. In fact, the heterologous expression of a Vitreoscilla sp. hemoglobin gene in $R$. etli led to an increase in respiratory activity in free-living rhizobia and

Table 1. Upregulated plant genes in nodules responsive to ReOtsA overexpression in Rhizobium etli

\begin{tabular}{|c|c|c|c|c|c|}
\hline \multirow[b]{2}{*}{ Annotation $^{a}$} & \multirow[b]{2}{*}{ Pathway } & \multicolumn{2}{|c|}{ Irrigated plants } & \multicolumn{2}{|c|}{ Drought-stressed plants } \\
\hline & & $\mathbf{O x} / \mathbf{W T}^{\mathbf{b}}$ & No. of EST & $O x / W T^{b}$ & No. of EST \\
\hline \multicolumn{6}{|l|}{ Nodulins and nitrogen metabolism } \\
\hline Leghemoglobin 2 & Oxygen transport & 7 & 38 & 37 & 209 \\
\hline Nodulin 30 & Unknown function & 7 & 15 & 31 & 149 \\
\hline Glutamine synthetase & $\mathrm{NH}_{4}$ assimilation & 2 & 1 & 11 & 23 \\
\hline Uricase II & Ureide synthesis & - & 0 & 7 & 15 \\
\hline Nitrate reductase & $\mathrm{NH}_{4}$ assimilation & 2 & 1 & 3 & 3 \\
\hline \multicolumn{6}{|l|}{ Carbon metabolism } \\
\hline Sucrose synthase & Sucrose cleavage & - & 0 & 6 & 17 \\
\hline Malate dehydrogenase & Malate synthesis & 2 & 1 & 5 & 10 \\
\hline PEP carboxylase & Oxaloacetate synthesis & 2 & 1 & 3 & 5 \\
\hline Trehalase & Trehalose cleavage & - & 0 & 3 & 3 \\
\hline \multicolumn{6}{|l|}{ Abiotic stress } \\
\hline Homoglutathione synthetase & Detoxification & 6 & 1 & 32 & 1 \\
\hline Catalase & Detoxification & - & 0 & 16 & 3 \\
\hline Cytochrome P450 & Detoxification & - & 0 & 10 & 12 \\
\hline Glutaredoxin & Detoxification & - & 0 & 9 & 6 \\
\hline Ascorbate peroxidase & Detoxification & - & 0 & 6 & 13 \\
\hline Heat-shock transcription factor & Heat tolerance & - & 0 & 6 & 3 \\
\hline $\mathrm{Zn} / \mathrm{Cu}$ superoxide dismutase & Detoxification & 2 & 1 & 4 & 3 \\
\hline Heat-shock 70 kDa protein & Heat tolerance & - & 0 & 4 & 1 \\
\hline $\mathrm{K}^{+} / \mathrm{Na}^{+}$symporter $\mathrm{HKT} 1$ & Salt tolerance & - & 0 & 3 & 1 \\
\hline Glutathion peroxidase & Detoxification & - & 0 & 2 & 1 \\
\hline
\end{tabular}

\footnotetext{
${ }^{a}$ Enlisted genes had an enhanced expression, as evidenced by macroarrays of all the corresponding expressed sequence tags (EST) spotted in the membrane.

${ }^{\mathrm{b}}$ Expression ratio. The EST's highest expression ratio is shown for the corresponding nodule gene of plants inoculated with $R$. etli overexpressing ReOtsA (Ox) and wild-type (WT) strains.
} 
higher nitrogenase activity and total nitrogen content in bean plants (Ramírez et al. 1999).

The macroarray data suggest that the possible signaling role of trehalose coordinates the gene expression of nitrogen metabolism genes such as glutamine synthetase and uricase II, with the carbon metabolism genes of sucrose synthase, PEP carboxylase, and malate dehydrogenase required to increase the availability of carbon skeletons to the bacteroid; concomitantly, stress-adaptive genes are expressed to remove free radicals or other toxic molecules and to help plants recover from drought stress. Upon drought stress, a significantly higher expression of all gene categories was observed in plants inoculated with the $R$. etli overexpressing OtsA (Table 1), supporting the role of trehalose in triggering stress response.

\section{Conclusion.}

The overexpression of ReOtsA confers tolerance to osmotic stress in free-living $R$. etli, providing the inoculant with a greater survival capacity and symbiotic efficiency. In plant nodules, the symbiotic bacteroid with higher trehalose content results in an increased nodulation and nitrogenase activity, which leads to a higher biomass and grain yield. Trehalose is a signal, possibly traveling from the bacteroid to the plant, that also triggers an enhanced drought tolerance. Thus, the overexpression of TPS in rhizobia establishes a new method to obtain a biofertilizer that could be used in agriculture to improve grain production and drought tolerance in legume crops.

\section{MATERIALS AND METHODS}

\section{Biological material and plant growth.}

$R$. etli CE3 strain and $P$. vulgaris var. Negro Jamapa plants were used in the present study. Inoculation of bean plants with $R$. etli WT, mutant, and overexpressing strains was done in the following way. Seed were surface sterilized with sodium hypochlorite $10 \%$ solution for $15 \mathrm{~min}$. After washing the seed with water, they were transferred to a tray containing paper towels moistened in water, and thereafter covered with aluminum foil to allow seed germination at $27^{\circ} \mathrm{C}$ during 72 h. After germination, each seedling planted in pots containing sterile vermiculite was inoculated with $1 \mathrm{ml}$ of $R$. etli culture. Plants were watered with Hoagland's solution (Sigma, St. Louis) every other day.

\section{Cloning $R$. etli OtsA gene and mutant isolation.}

To clone the OtsA gene from $R$. etli, two oligonucleotides (forward, 5'-ATCATCGGTGTCGATCGCCT and reverse, 5'GGATCGTCCGGATCCTGC) were designed to flank a conserved region from the OtsA gene from Mesorhizobium loti, S. meliloti, and Rhizobium sp. NGR234, and were used to amplify a DNA fragment by PCR $\left(1\right.$ cycle at $94^{\circ} \mathrm{C}$ for $3 \mathrm{~min}$; 30 cycles at $94^{\circ} \mathrm{C}$ for $1 \mathrm{~min}, 60^{\circ} \mathrm{C}$ for $2 \mathrm{~min}$, and $72^{\circ} \mathrm{C}$ for 3 min; and 1 cycle at $72^{\circ} \mathrm{C}$ for $10 \mathrm{~min}$ ) using as a template the $R$. etli genome. The resulting 370-bp fragment was cloned in PCR2.1-TOPO vector (Invitrogen, Carlsbad, CA, U.S.A.) and sequenced. For $R$. etli mutagenesis by homologous recombination, E. coli S17-1 strain hosting the pSUP202 suicide vector (Simon et al. 1983), which had the ReOtsA 370-bp fragment, was used. Two $R$. etli mutants were obtained after selecting in tetracycline, which were characterized by Southern blot and sequencing. To clone the full-length OtsA gene from $R$. etli, a genomic library from this species was constructed in pBluescript (Stratagene, La Jolla, CA, U.S.A.). After library screening, four clones hybridizing to the 370-bp probe were isolated. Sequencing both ends of each clone showed that the gene was cloned in parts. To obtain the full-length codingsequence, forward (5'-CCGCTCGAGAAGGAAAACCCCAT
GAGCCGTCTTATC, which contains a XhoI site and the $R$. etli ribosome binding site; the initiation codon is underlined) and reverse (5'-CCCAAGCTTAGCGCGCTAAAGAGCCTA TCCGTG, which contains a HindIII site; a STOP codon is underlined) oligonucleotides were used to amplify it by PCR ( 1 cycle at $94^{\circ} \mathrm{C}$ for $5 \mathrm{~min} ; 30$ cycles at $94^{\circ} \mathrm{C}$ for $1.5 \mathrm{~min}$, $55^{\circ} \mathrm{C}$ for $1 \mathrm{~min}$, and $72^{\circ} \mathrm{C}$ for $1.75 \mathrm{~min}$; and 1 cycle at $72^{\circ} \mathrm{C}$ for $10 \mathrm{~min}$ ) using $R$. etli genome as a template. A predicted 1.4-kb fragment was obtained. The ReOtsA gene was then subcloned in the broad-host-range pBBR1MCS5 expression vector (Kovach et al. 1995) after transformation of E. coli DH5 $\alpha$ strain. The resulting plasmid was named pBBR1M::ReOtsA and was used to transform E. coli S17-1 strain and mobilized to $R$. etli by conjugation.

\section{Stress tolerance tests in $R$. etli.}

Rhizobia were grown at $30^{\circ} \mathrm{C}$ in PY media supplemented with nalidixic acid $(20 \mu \mathrm{g} / \mathrm{ml})$ for the WT, tetracycline at 5 $\mu \mathrm{g} / \mathrm{ml}$ for the ots $A^{-}$mutant, and gentamycin at $30 \mu \mathrm{g} / \mathrm{ml}$ for the Ox strain. Precultures were spun down to wash the antibiotics and grown up to midlogarithmic phase (optical density of 0.4 at $600 \mathrm{~nm}$ ) in minimal medium supplemented with $0.4 \%$ glycerol. To determine survival upon osmotic stress, cells were cultured in $0.5 \mathrm{M} \mathrm{NaCl}$ for 2 days, and dilutions were made and plated on PY agar medium with its corresponding antibiotic at $30^{\circ} \mathrm{C}$ for $72 \mathrm{~h}$. After this time, the surviving colonies were counted.

\section{Drought tolerance tests and biomass determination in bean plants.}

Bean plants inoculated with the $R$. etli $\mathrm{WT}$, ots $A^{-}$mutant or Ox strains were subjected to drought stress for 3 weeks by stopping watering after 21 days of growth, and observed for wilting symptoms. Then, watering was reestablished for 1 day and photographed. To determine plant biomass, leaves were weighed immediately after detaching (fresh weight) and then placed in an oven at $70^{\circ} \mathrm{C}$ for $72 \mathrm{~h}$ before weighing again (dry weight). Relative water content was determined as reported before (Gaxiola et al. 2001).

\section{Trehalose quantification.}

To quantify the trehalose content in $R$. etli and bean plants, a standard protocol was used (Avonce et al. 2004). For bacteria, cultures were centrifuged, washed with water, and resuspended in $80 \%$ ethanol. After incubation at $85^{\circ} \mathrm{C}$ for $15 \mathrm{~min}$, the samples were centrifuged and the supernatant recovered. The ethanol excess was evaporated and samples were resuspended in ultrapure water before being analyzed by high-performance liquid chromatography (HPLC) using a ZORBAX carbohydrate analysis column (Agilent Technologies, Santa Clara, CA, U.S.A.) eluted with acetonitrile/water (65:35, vol/vol). To quantify trehalose from $P$. vulgaris, tissues were weighed and frozen in liquid nitrogen, resuspended in water, and boiled before being deionized in a 1-ml column prepared with cationic and anionic resins (Avonce et al. 2004) and then analyzed by HPLC as described before.

\section{Determination of nitrogenase activity.}

Root nodules were counted, separated, and used to determine enzyme activity (Wacek and Brill 1976). Nitrogenasespecific activity was determined in detached 21-day postinoculated nodulated roots by the acetylene-reduction activity in closed jars and were expressed as nanomoles of ethylene per minute per gram of nodule (fresh weight). Thirty min after the air was extracted and replaced with acetylene, a sample was injected in a gas chromatographer. After the assay, the nodule weight sample were determined. 


\section{Microscopy.}

Nodules from 21-day-old plants were harvested, rapidly fixed, and processed as reported (Trepp et al. 1999). The embedded tissues were sectioned in $7-\mu \mathrm{m}$ cuttings and placed in microscope slides covered with poly-L-lys. After paraffin elimination with xylene, the cuttings were stained in methylene blue solution, dehydrated, and mounted with Permount (Fisher Scientific, Pittsburgh). Sections were examined under a direct light microscope and photographed with a digital camera.

\section{Yield quantification in bean plants.}

Bean plants were inoculated with the $R$. etli WT, ots $A^{-}$mutant, or Ox strains, and had continual watering for 4 months until pods appeared. In order to determine the yield of inoculated plants with the different $R$. etli strains, the following parameters were taken: the number of pods and the number of seed on each pod were counted, and each seed was weighed. Yield $=$ (number of pods per plant) $\times$ (number of seed per pod $) \times($ seed weight) (Linkemer et al. 1998). Three independent experiments were carried out with three repetitions each time.

\section{Macroarray analysis of nodule genes.}

The EST sequences and nylon filter arrays were previously reported (Ramírez et al. 2005) and a full description of these data is available online. Total RNA was isolated from mature nodules 21 dpi from plants grown under drought stress or in normal conditions. Nodules were elicited with the $R$. etli WT, ots $A^{-}$mutant, or Ox strain. cDNA was in vitro synthesized from each RNA preparation and was radioactively labeled before using it as a probe to hybridize nylon membranes printed with $P$. vulgaris nodule EST as described before (Ramírez et al. 2005). The hybridization of macroarrays was repeated twice from each condition. The radioactivity of each spot was quantified using a Phosphor Screen imaging system (Molecular Dynamics, Sunnyvale, CA, U.S.A.) and the signal intensity was determined using the Array-Pro Analyzer (Media Cybernetics, Silver Spring, MD, U.S.A.) with normalization against background. Gene expression was considered reliably expressed if they showed an intensity/background ratio greater than 2 throughout all related parallel hybridizations.

\section{Statistical analysis.}

The data were processed by analysis of variance by $t$ Student test followed by Duncan-Waller mean analysis.

\section{ACKNOWLEDGMENTS}

We thank S. I. Fuentes for histological and microscopy analysis (Centro de Ciencias Genómicas-Universidad Nacional Autónoma de México [UNAM]), and P. Gaytán and E. López (Instituto de BiotecnologíaUNAM, Cuernavaca, Mexico) for oligonucleotide synthesis. This work was supported by CYTED grant 107PIC0312 and CONACYT grant 2004CO1-46078 (to G. Iturriaga).

\section{LITERATURE CITED}

Aeschbacher, R. A., Müller, J., Boller, T., and Wiemken, A. 1999. Purification of the trehalase GMTRE1 from soybean nodules and cloning of its cDNA. GMTRE1 is expressed at a low level in multiple tissues. Plant Physiol. 119:489-495.

Avonce, N., Leyman, B., Mascorro-Gallardo, J. O., Van Dijck, P., Thevelein, J. M., and Iturriaga, G. 2004. The Arabidopsis trehalose-6-P synthase AtTPS1 gene is a regulator of glucose, abscisic acid, and stress signaling. Plant Physiol. 136:3649-3659.

Avonce, N., Mendoza-Vargas, A., Morett, E., and Iturriaga, G. 2006. Insights on the evolution of trehalose biosynthesis. BMC Evol. Biol. 6:109.

Bartels, D., and Sunkar, R. 2005. Drought and salt tolerance in plants. Crit. Rev. Plant Sci. 24:23-58.

Bray, E. A., Bailey-Serres, J., and Weretilnyk, E. 2000. Pages 1158-1249 in: Biochemistry and Molecular Biology of Plants. B. B. Buchanan, W.
Gruissem, and R. Jones, eds. American Society of Plant Physiologists, Rockville, MD, U.S.A.

Brockwell, J., Bottomley, P. J., and Thiers, J. E. 1995. Manipulation of rhizobia microflora for improving legume productivity and soil fertility: A critical assessment. Plant Soil 174:143-180.

Clegg, J. S. 2001. Cryptobiosis-a peculiar state of biological organization Comp. Biochem. Physiol. 128:613-624.

Crowe, J. H., Hoekstra, F. A., and Crowe, L. M. 1992. Anhydrobiosis. Annu. Rev. Physiol. 54:579-599.

Cytryn, E. J., Sangurdekar, D. P., Streeter, J. G., Franck, W. L., Chang, W.-K., Satcey, G., Emerich, D. W., Joshi, T, Xu, D., and Sadowsky, M. J. 2007. Transcriptional and physiological responses of Bradyrhizobium japonicum to desiccation-induced stress. J. Bacteriol. 189:6751-6762.

Eastmond, P. J., van Dijken, A. J. H., Spielman, M., Kerr, A., Tissier, A. F., Dickinson, H. G., Jones, J. D. G., Smekeens, S. C., and Graham, I. A. 2002. Trehalose-6-phosphate synthase 1, which catalyses the first step in trehalose synthesis, is essential for Arabidopsis embryo maturation. Plant J. 29:225-235.

Elbein, A. D., Pan, Y. T., Pastuszak, I., and Carroll, D. 2003. New insights on trehalose: A multifunctional molecule. Glycobiolgoy 13:17R-27R.

Foster, A. J., Jenkinson, J. M., and Talbot, N. J. 2003. Trehalose synthesis and metabolism are required at different stages of plant infection by Magnaporthe grisea. EMBO (Eur. Mol. Biol. Organ.) J. 22:225-235.

Gaff, D. F. 1996. Tobacco-plant desiccation tolerance. Nature 382:502.

Garg, A. K., Kim, J.-K., Owens, T. G., Ranwala, A. P, Choi, Y. D., Kochian, L. V., and Wu, R. J. 2002. Trehalose accumulation in rice plants confers high tolerance levels to different abiotic stresses. Proc. Natl. Acad. Sci. U.S.A. 25:15898-15903.

Gaxiola, R. A., Li, J., Undurraga, S., Dang, L. M., Allen, G. J., Alper, S. L., and Fink, G. R. 2001. Drought-and salt-tolerant plants result from overexpression of the AVP1 $\mathrm{H}^{+}$-pump. Proc. Natl. Acad. Sci. U.S.A. 98:11444-11449.

Giaever, H. M., Styrvold, O. B., Kaasen, I., and Ström, A. R. 1988. Biochemical and genetic characterization of osmoregulatory trehalose synthesis in Escherichia coli. J. Bacteriol. 170:2841-2849.

González, V., Santamaría, R. I., Bustos, P., Hernández-González, I., Medrano-Soto, A., Moreno-Hagelsieb, G., Janga, S. C., Ramírez, M. A., Jimenez-Jacinto, V., Collado-Vides, J., and Davila, G. 2006. The partitioned Rhizobium etli genome: Genetic and metabolic redundancy in seven interacting replicons. Proc. Natl. Acad. Sci. U.S.A. 103:3834-3839.

Gonzalez-Parraga, P., Hernandez, J. A., and Arguelles, J. C. 2003. Role of antioxidant enzymatic defences against oxidative stress $\mathrm{H}_{2} \mathrm{O}_{2}$ and the acquisition of oxidative tolerance in Candida albicans. Yeast 20:11611169.

Guo, N., Puhlev, I., Brown, D.R., Mansbridge, J., and Levine, F. 2000. Trehalose expression confers desiccation tolerance on human cells. Nat. Biotechnol. 18:168-171.

Holmström, K.-O., Mäntylä, E., Welin, B., Mandal, A., Palva, E. T., Tunnela, O. E., and Londesborough, J. 1996. Drought tolerance in tobacco. Nature 379:683-684.

Jensen, J. B., Ampomah, O. Y., Darrah, R., Peters, N. K., and Bhuvaneswari, T. V. 2005. Role of trehalose transport and utilization in Sinorhizobium meliloti-alfalfa interactions. Mol. Plant-Microbe Interact. 18:694-702.

Kandror, O., De Leon, A., and Goldberg, A. L. 2002. Trehalose synthesis is induced upon exposure of Escherichia coli to cold and is essential for viability at low temperatures. Proc. Natl. Acad. Sci. U.S.A. 99:92279732.

Kovach, M. E., Phillips, R. W., Elzer, P. H., Roop, R. M., and Peterson, K. M. 1995. Four new derivatives of the broad-host-range cloning vector pBBR1MCS, carrying different antibiotic-resistance cassettes. Gene 166:175-176.

Linkemer, G., Board, J. E., and Musgrave, M. E. 1998. Waterlogging effects on growth and yield components in late-planted soybean. Crop. Sci. 38:1576-1584.

Müller, J., Boller, T., and Wiemkem, A. 2001. Trehalose becomes the most abundant non-structural carbohydrate during senescence of soybean nodules. J. Exp. Bot. 52:943-947.

Murphy, H., Graham, R., Vladimir, V., and Robertson, B. 2005. The OtsAB pathway is essential for trehalose biosynthesis in Mycobacterium tuberculosis. J. Biol. Chem. 280:14524-14529.

Paul, M. 2007. Trehalose 6-phosphate. Curr. Opin. Plant Biol. 10:303-309.

Purvis, J. E., Yomano, L. P., and Ingram, L. O. 2005. Enhanced trehalose production improves growth of Escherichia coli under osmotic stress. Appl. Environ. Microbiol. 71:3761-3769.

Ramírez, M., Valderrama, B., Arredondo-Peter, R., Soberón, M., Mora, J., and Hernández, G. 1999. Rhizobium etli genetically engineered for the heterologous expression of Vitreoscilla sp. hemoglobin: Effects on freeliving and symbiosis. Mol. Plant-Microbe Interact. 12:1008-1015. 
Ramírez, M., Graham, M. A., Blanco-Lopez, L., Silvente, S., MedranoSoto, A., Blair, M. W., Hernandez, G., Vance, C. P., and Lara, M. 2005. Sequencing and analysis of common bean ESTs. Building a foundation for functional genomics. Plant Physiol. 137:1211-1227.

Rolland, F., Baena-Gonzalez, E., and Sheen, J. 2006. Sugar sensing and signaling in plants: Conserved and novel mechanisms. Annu. Rev. Plant Biol. 57:675-709.

Satoh-Nagasawa, N., Nagasawa, N., Malcomber, S., Sakai, H., and Jackson, D. 2006. A trehalose metabolic enzyme controls inflorescence architecture in maize. Nature 441:227-230.

Schluepmann, H., Pellny, T., van Dijken, A., Smeekens, S., and Matthew, P. 2003. Trehalose 6-phosphate is indispensable for carbohydrate utilization and growth in Arabidopsis thaliana. Proc. Natl. Acad. Sci. U.S.A. 100:6849-6854.

Schluepmann, H., van Dijken, A., Aghdasi, M., Wobbes, B., Paul, M., and Smeekens, S. 2004. Trehalose mediated growth inhibition of Arabidopsis seedlings is due to trehalose-6-phosphate accumulation. Plant Physiol. 135:879-890.

Sessitsch, A., Howieson, J. G., Perret, X., Antoun, H., and MartínezRomero, E. 2002. Advances in Rhizobium research. Crit. Rev. Plant Sci. 21:323-378

Simon, R., Priefer, U., and Pühler, A. 1983. A broad host range mobilization system for in vivo genetic engineering: Transposon mutagenesis in gram negative bacteria. Biotechnology 1:784-791.

Socolow, R. H. 1999. Nitrogen management and the future of food: Lessons from the management of energy and carbon. Proc. Natl. Acad. Sci. U.S.A. 96:6001-6008

Streeter, J. G. 1985. Accumulation of alpha,alpha-trehalose by Rhizobium bacteria and bacteroids. J. Bacteriol. 164:78-84

Streeter, J. G. 2003. Effect of trehalose on survival of Bradyrhizobium japonicum during desiccation. J. Appl. Microbiol. 95:484-491.

Ström, A. R., and Kaasen, I. 1993. Trehalose metabolism in Escherichia coli: Stress protection and stress regulation of gene expression. Mol. Microbiol. 8:205-210.

Subbarao, G. V., Johansen, C., Slinkard, A. E., Nageswara Rao, R. C., Saxena, N. P., and Chauhan, Y. S. 1995. Strategies for improving drought resistance in grain legumes. Crit. Rev. Plant Sci. 14:469-523.

Trepp, G. B., Plank, D. W., Gantt, J. S., and Vance, C. P. 1999. NADHGlutamate synthase in alfalfa root nodules. Immunocytochemical localization. Plant Physiol. 119:829-837.

Wacek, T., and Brill, W. J. 1976. Simple, rapid assay for screening nitrogen fixing ability in soybean. Crop Sci. 16:519-522.

Wilson, R.A., Jenkinson, J. M., Gibson, R. P., Littlechild, J. A., Wang, Z Y., and Talbot, N. J. 2007. Tps1 regulates the pentose phosphate pathway, nitrogen metabolism and fungal virulence. EMBO (Eur. Mol. Biol. Organ.) J. 26:3673-3685.

Yeo, E. T., Kwon, H. B., Han, S. E., Lee, J. T., Ryu, J. C., and Byun, M. 2000. Genetic engineering of drought resistant potato plants by introduction of the trehalose-6-phosphate synthase TPS1 gene from Saccharomyces cerevisiae. Mol. Cell 10:263-263.

\section{AUTHOR-RECOMMENDED INTERNET RESOURCE}

Center for Genomic Sciences (CCG) Phaseolus vulgaris EST Project: www.ccg.unam.mx/phaseolusest/Data_download.htm 